\section{Visual results in children treated for macular retinoblastoma}

\section{Abstract}

Purpose To evaluate the results of patching treatment in children with macular retinoblastoma in one eye.

Methods Fifteen children affected by macular retinoblastoma received instructions for patching treatment for amblyopia. Data were collected on age at diagnosis of the tumor, presence of unilateral or bilateral disease, area of posterior pole involvement by the scar of the regressed tumor and its relationship to the fovea; and the onset, duration, and compliance of patching. The visual acuities recorded were expressed in logMAR (logarithm minimum angle of resolution) equivalents.

Results Twelve children $(80 \%)$ had bilateral retinoblastoma with the macular involved in one eye and three children had unilateral macular tumors. The median age at which patching was initiated was 15 months (range 4-36). Compliance to patching was good in $80 \%$ of children, with a median duration of $4 \mathrm{~h}$ (range $0.5-8$ ) per day, 7 days per week, with total occlusion of the better eye. The median percentage of posterior pole involvement was 34\% (range 11-100\%). Eighty percent of children had some improvement in their visual acuity, and of the children in whom final $\log$ MAR acuity was recorded, $73 \%$ had an acuity of $\mathbf{1 . 0}$ $\log$ MAR or better and $53 \%$ an acuity of 0.5 $\log$ MAR or better after patching. There was no evidence of association between age of patient, sex, duration of patching, or percentage of posterior pole involvement and the improvement in visual acuity.

Conclusions In spite of the macular involvement of eyes with retinoblastoma, some visual recovery was achieved in $80 \%$ of children. Hence a trial of patching therapy is recommended for all children with involvement of the macula by retinoblastoma.
P Watts ${ }^{1,3}$, C Westall 1,3, L Colpa ${ }^{1,3}$, L MacKeen $^{1}$ M Abdolell ${ }^{2,4}$, B Gallie ${ }^{1,3}$ and E Héon ${ }^{1,3}$

Eye (2002) 16, 75-80. DOI: 10.1038/

sj/EYE/6700070

Keywords: amblyopia; retinoblastoma; visual acuity

\section{Introduction}

The primary goal in the management of retinoblastoma is preservation of life. With current survival rates of $92-96 \%,{ }^{1,2}$ attention can also be focused on preserving the affected eye. ${ }^{3}$ The eye can be saved by various combinations of therapy, including focal therapy, chemotherapy, and radiotherapy. ${ }^{4-6}$ The rate of success depends on the severity of involvement, and the visual potential on the size and location of the tumors. ${ }^{1}$ Current protocols for chemotherapy increase the likelihood of eyes being saved. ${ }^{4}$ The visual acuities of the eyes surviving after treatment of macular tumors have varied from 20/20 to no light perception. ${ }^{3,7-9}$ The eyes showing excellent visual results in these reports were the only remaining eyes the children had; hence amblyopia was not an issue.

No study to date has focused on the management of associated amblyopia in patients with retinoblastoma. Though involvement of the fovea by the tumor indicates a poor visual outcome, it is not possible to predict the visual acuity accurately. ${ }^{9}$ A report documenting visual recovery with amblyopia therapy in a child with foveal involvement suggests that the amblyopia is superimposed on organic ocular disease. ${ }^{10}$ Visual recovery after occlusion therapy in children with structural abnormalities of the optic disc and macula has also been reported. ${ }^{11}$ These and other findings suggest reversible amblyopia may coincide with visual loss due to an underlying structural abnormality. ${ }^{11,12}$
'Department of Ophthalmology

The Hospital for Sick Children

Toronto, Ontario, Canada

2Department of Population Health Sciences

The Hospital for Sick Children

Toronto, Ontario, Canada

${ }^{3}$ Department of Ophthalmology

The University of Toronto Toronto, Ontario, Canada

${ }^{4}$ Department of Public Health Sciences The University of Toronto Toronto, Ontario, Canada

Correspondence:

$P$ Watts

49 St Agatha Road Cardiff CF14 4EA, UK

Tel: 0292020591

Fax: 02920610746 hotmail.com
E-mail: patrickowatts@ 
Current practice is to assess visual acuity in all retinoblastoma patients using preferential looking techniques and to initiate occlusion therapy when there is evidence of difference in acuity greater than 0.2 logMAR between the eyes. We report that occlusion therapy can improve visual acuity of eyes affected by macular retinoblastoma.

\section{Patients and methods}

To be eligible for this study, patients had two eyes surviving bilateral retinoblastoma with macular involvement in at least one of them, or eyes surviving unilateral macular retinoblastoma. Cases were ascertained from the retinoblastoma and orthoptic database. The case notes of all children with bilateral retinoblastoma and those with unilateral retinoblastoma that were not enucleated were hand searched to see if they met the above criteria. All children had visual acuity assessment before, and two or more consecutive assessments after initiation of patching. A retrospective chart review was carried out to record demographic data, age at presentation, treatment received, patching regimen and longitudinal visual acuity data.

Parents were encouraged to patch the good eye maximally depending on the age of the child. The patching regimen was part-time total occlusion, from 4-8 hours a day.

Initial and final digital images acquired by the Retcam (Massie Research Laboratories, Dublin, CA, USA) were reviewed to check the extent of macular involvement. Macular tumors were defined as those located within 1.5 disc diameters of the fovea. Foveal involvement was judged by indirect ophthalmoscope examination or by examination of the Retcam digital images. For the purposes of this study, the macular area was determined by drawing a circle centered on the macula with a radius equal to the distance between this point and the temporal edge of the optic disc. The area within this circle that was occupied by the tumor was used to calculate the percentage of macular involvement (Figure 1).

Patients who continued to be followed at our hospital were given a final visual acuity assessment at the time of the study. Longitudinal data were recorded at 3- to 6- month intervals at our institution and from the local eye care provider in the patients being followed at other institutions. Visual acuities were recorded with Cardiff vanishing optotypes (Keeler, Windsor, UK), Teller cards (Vistech Consultants, Dayton, OH, USA), or linear Snellen acuity, then converted to $\log$ MAR values. Recorded acuities were

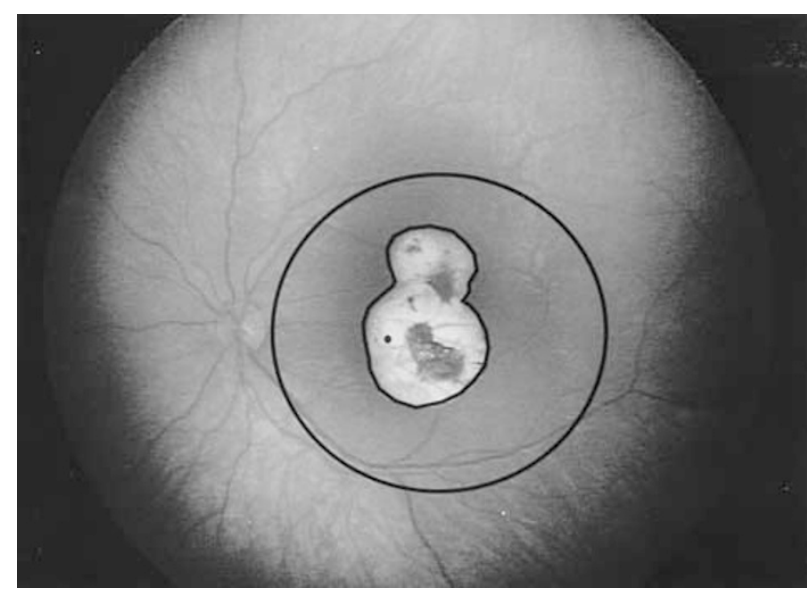

Figure 1 Area of macular scar, from treated retinoblastoma. Scar outlined within the large circle superimposed on the posterior pole. The black dot signifies the calculated centre of the fovea. Patient 3 (Table 1), initial vision before patching 1 logMAR, final vision following patching $0.1 \log$ MAR.

obtained with the same testing method on all occasions for each child.

Visual acuity improvement from patching was considered to be the difference between total visual acuity improvement in the affected eye, including the effect of maturation and patching (final logMAR acuity - baseline logMAR acuity of the involved eye), and visual acuity improvement due to a maturational effect (final $\log$ MAR acuity - baseline $\log$ MAR acuity of the better eye). Compliance with patching was recorded as good or poor depending on the number of hours prescribed and the number carried out. Good compliance was defined as patching equal to or greater than $50 \%$ and poor compliance less than $50 \%$ of the prescribed hours, as reported by parents.

Association between patching, location of tumor, and visual outcome was examined using the chi-square test. Analysis of covariance (ANCOVA) was used to assess the effect of age and visual improvement. A repeated measures analysis was conducted of the initial and final $\log$ MAR values using a two-factor (macular involvement and time of logMAR estimates) experimental design with a repeated measure of the time factor. Finally, a multivariate analysis of covariance was conducted to investigate the effect of covariates such as age at diagnosis and the percentage of macular involvement on the primary repeated measures analysis.

\section{Results}

Fifteen children were included in this study. Twelve children had bilateral tumors with macular involvement in at least one eye, and three had 
unilateral macular tumors. There were eight male patients. Median age at time of diagnosis was 5 months (range -2 to 30 ). One child (patient 1) was diagnosed with a macular tumor at 33 weeks' gestation by obstetrical ultrasound. Median age of the children at initiation of patching was 15 months (range 4-36) (Table 1). The median follow-up period was 25 months with a range of 10 months to 7 years.

Thirteen patients were treated with combined chemotherapy and focal therapy, and two received additional radiotherapy (patient 1: stereotactic radiotherapy and patient 13: external beam therapy; Table 1). Details of the protocols have been published previously. ${ }^{4}$ All patients received focal therapy, consisting of laser therapy alone (two patients) or a combination of laser and cryotherapy. The duration of patching ranged from 0.5 to 8 hours/day, with a median period of 4 hours/day.

Foveal involvement did not predict response to patching $(P=0.49)$. In fact, some patients with foveal involvement improved with patching (Figure 2). No significant correlation was found between visual improvement and number of hours that patching was carried out $(P=0.285)$, as there were only three patients whose compliance was poor (patients 12, 13 and 15: Table 1). There was no association between age at diagnosis or at initiation of patching and the final visual outcome.

In the macular-scarred eyes, the initial visual acuity ranged from unrecordable to $0.9 \log \mathrm{MAR}$, and final visual acuities from unrecordable to $0.1 \log$ MAR. Eleven of the 15 children $(73.3 \%)$ had a final visual acuity of $1.0 \log$ MAR or better, and eight of the 15 (53.3\%) achieved 0.5 logMAR or better following occlusion therapy. Three children had a visual acuity between $1.1 \log$ MAR and hand movements, and 1 child had no recordable visual acuity at the final visit following patching.

Five patients had strabismus (four exotropia and one esotropia). Four of these patients (patients 3, 5, 8 (esotropia) and 11) showed an improvement in visual acuity with patching, ranging from 0.1 to $0.7 \log$ MAR. One child (Table 1, patient 14) improved to hand movement vision from an initial vision of light perception.

The vision recorded was better at final than initial testing in $80 \%$ of children. Ten of the 11 children (90.9\%) in whom logMAR acuities could be recorded showed improvement in visual acuity, with a mean difference between baseline and final score recorded as $0.67 \log$ MAR difference $(P=0.0006)$ (Figure 3$)$. At baseline the $\log$ MAR difference between the fellow and macular involved eyes was 0.58 and the final $\log$ MAR difference was 0.28 . The 0.3 decrease in the difference from baseline to final scores represents improvement in visual acuity of the macular-scarred eyes.

For some children patching was initiated during the period of visual maturation; hence some of the improvement noted during the course of patching could be simply a maturational effect. Figure 4 shows the improvement of vision by patching over and above the effect of maturation in the 11 children in whom it was possible to record logMAR acuity.

Eight of the eleven children $(73 \%)$ with logMAR

Table 1 Demographic data of children with macular retinoblastoma

\begin{tabular}{|c|c|c|c|c|c|c|c|c|c|c|c|c|c|c|}
\hline \multirow[t]{3}{*}{ Pt } & \multirow{3}{*}{ Sex } & \multirow{3}{*}{$\begin{array}{c}\text { Bilateral } \\
v s \\
\text { unilateral }\end{array}$} & \multirow{3}{*}{$\begin{array}{l}\text { Age at } \\
\text { diagnosis } \\
\text { (months) }\end{array}$} & \multirow{3}{*}{$\begin{array}{l}\text { Age of } \\
\text { onset of } \\
\text { patching } \\
\text { (months) }\end{array}$} & \multirow{3}{*}{$\begin{array}{l}\text { Age at } \\
\text { final } \\
\text { acuity }\end{array}$} & \multirow{3}{*}{$\begin{array}{c}\text { Duration } \\
\text { of } \\
\text { patching } \\
\text { (months) }\end{array}$} & \multirow{3}{*}{$\begin{array}{l}\text { Patching } \\
\text { hours/day }\end{array}$} & \multirow{3}{*}{$\begin{array}{c}\text { Macular } \\
\text { involvement } \\
(\%)\end{array}$} & \multirow{3}{*}{$\begin{array}{l}\text { Foveal } \\
\text { involvement }\end{array}$} & \multirow{3}{*}{$\begin{array}{l}\text { Compliance } \\
\text { with } \\
\text { patching }\end{array}$} & \multicolumn{4}{|c|}{ Visual acuity (logMAR) } \\
\hline & & & & & & & & & & & \multicolumn{2}{|c|}{$\begin{array}{l}\text { Macular } \\
\text { tumor eye }\end{array}$} & \multicolumn{2}{|c|}{ Control eye } \\
\hline & & & & & & & & & & & Initial & Final & Initial & Final \\
\hline 1 & f & bilateral & -2 & 4 & 12 & 8 & 2 & 58 & yes & good & 1.4 & 1.0 & 0.6 & 0.5 \\
\hline 2 & f $\quad$ l & bilateral & 0.03 & 9 & 13 & 4 & 4 & 34 & no & good & 1.4 & 0.5 & 0.7 & 0.2 \\
\hline 3 & $\mathrm{~m}$ & bilateral & 0.3 & 18 & 31 & 13 & 5 & 20 & yes & good & 1.0 & 0.1 & 0.2 & 0.0 \\
\hline 4 & f 1 & bilateral & 0.7 & 35 & 85 & 50 & 8 & 28 & no & good & 1.0 & 0.3 & 0.0 & 0.0 \\
\hline 5 & $\mathrm{~m}$ & unilateral & 1 & 15 & 34 & 19 & 4 & 11 & yes & good & 1.0 & 0.5 & 0.8 & 0.0 \\
\hline 6 & f $\quad$ l & bilateral & 2 & 12 & 27 & 15 & 4 & 60 & yes & good & 0.9 & 0.5 & 0.7 & 0.1 \\
\hline 7 & $\mathrm{~m}$ & bilateral & 5 & 8 & 15 & 7 & 4 & 11 & no & good & 1.1 & 1.1 & 0.9 & 1.2 \\
\hline 8 & $\mathrm{~m}$ & bilateral & 5 & 14 & 80 & 66 & 4 & 19 & yes & good & 1.0 & 0.5 & 0.3 & 0.3 \\
\hline 9 & $\mathrm{~m}$ & bilateral & 6 & 12 & 32 & 20 & 4 & 16 & yes & good & 0.9 & 0.3 & 0.7 & 0.2 \\
\hline 10 & f $\quad$ l & bilateral & 9 & 24 & 53 & 29 & 5 & 19 & no & good & 2.1 & 0.4 & 1.6 & 0.3 \\
\hline 11 & $\mathrm{~m}$ & unilateral & 11 & 15 & 21 & 6 & 5 & 84 & yes & good & 1.2 & 0.7 & 0.2 & 0.0 \\
\hline 12 & f $\quad$ l & bilateral & 3 & 9 & 16 & 7 & 0.5 & 71 & yes & poor & $\mathrm{U}$ & $\mathrm{U}$ & 0.6 & 0.4 \\
\hline 13 & $\mathrm{~m}$ & bilateral & 30 & 36 & 42 & 6 & 1 & 82 & yes & poor & $\mathrm{U}$ & 1 & 0.3 & 0.3 \\
\hline 14 & & unilateral & 12 & 24 & 50 & 26 & 4 & 89 & yes & good & LP & $\mathrm{HM}$ & 0.3 & 0.3 \\
\hline 15 & $\mathrm{~m} \mathrm{l}$ & bilateral & 10 & 20 & 58 & 38 & 1 & 100 & yes & poor & $\mathrm{U}$ & $\mathrm{CF} 1 \mathrm{ft}$ & 0.3 & 0 \\
\hline
\end{tabular}

$\mathrm{f}=$ female, $\mathrm{m}=$ male, $\mathrm{U}=$ unrecordable, $\mathrm{CF}=$ counting fingers, $\mathrm{HM}=$ hand movements, $\mathrm{LP}=$ light perception, $\mathrm{ft}=$ foot. 

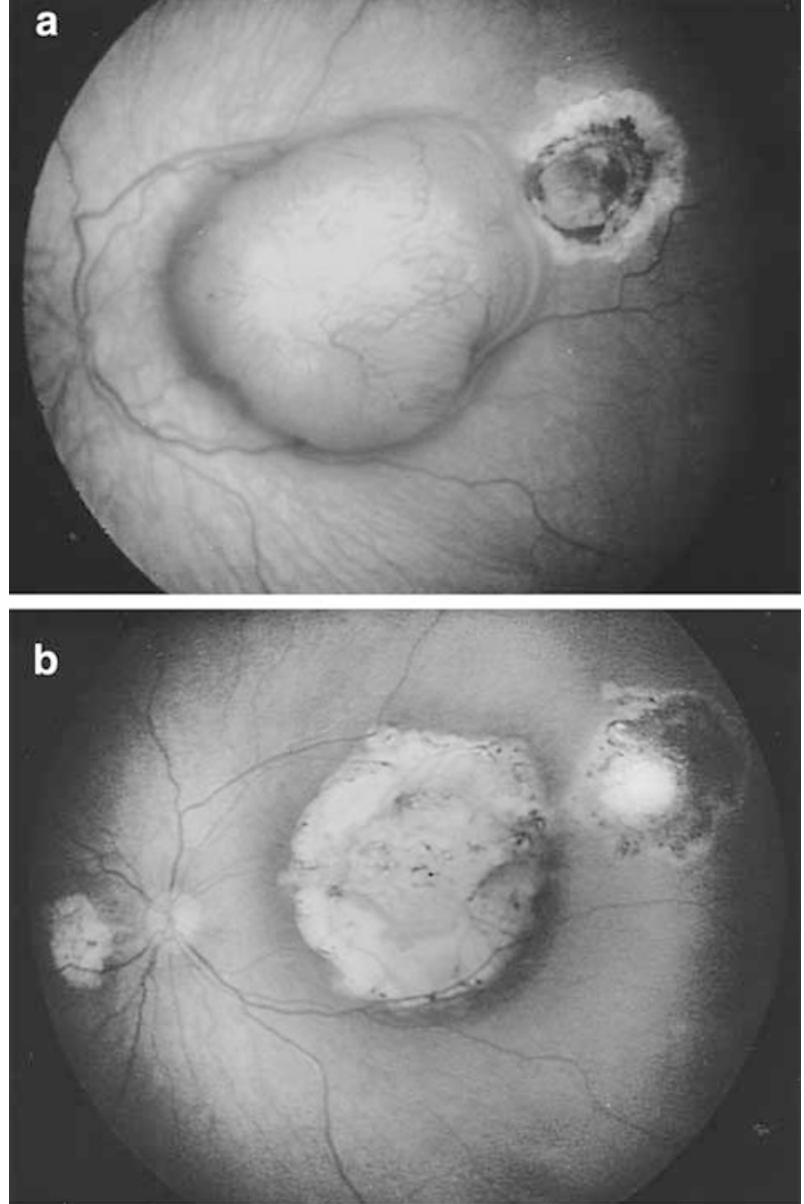

Figure 2 (a) Initial appearance of macular tumor of patient 6 (Table 1), 2 weeks after the initiation of chemotherapy and focal therapy, and (b) final appearance of macular scar final visual acuity. Initial vision before patching $0.9 \log \mathrm{MAR}$, final vision following patching $0.5 \log$ MAR.

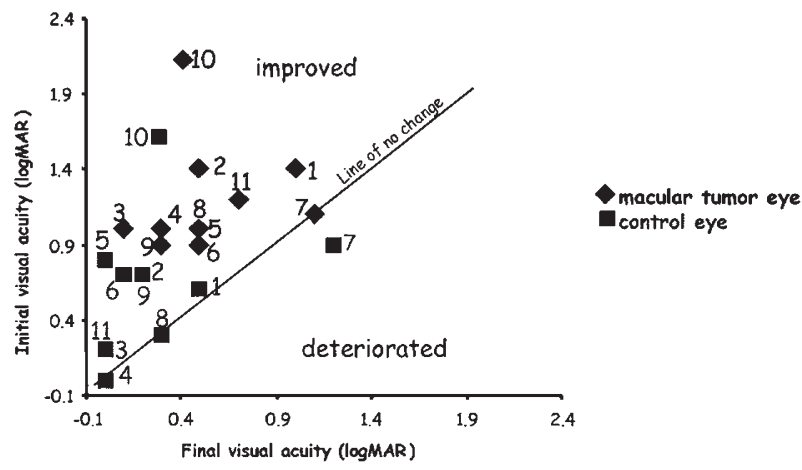

Figure 3 Graph demonstrating visual improvement with patching in macula-scarred eyes. The maturational effect of the control eyes seen in all eyes except patient 7 was there was a deterioration of vision in the control eye with patching. acuity scores demonstrated an improvement above the possible effect of maturation. These results must be interpreted with caution in view of the small numbers of subjects involved.

\section{Discussion}

Visual acuity improved with patching in the macular scarred eyes in $73 \%(8 / 11)$ of children for whom $\log$ MAR visual acuities could be recorded initially, and if gross visual responses are included, 80\% (12/15) showed some improvement. Visual acuity improvement in eyes affected by macular retinoblastoma may result from a decrease in the coexisting amblyopia or from natural visual maturation.

In a study ${ }^{13}$ of amblyopia untreated owing to lack of compliance (equivalent to the natural history of amblyopia), $41 \%$ of patients showed a deterioration of visual acuity over time, with no apparent maturational effect. In addition, Weiss et $a l^{8}$ reported that improvement in visual acuity regressed in a patient with macular retinoblastoma who discontinued amblyopia therapy. These studies suggest that the visual acuity in the macular-scarred eyes in our study group might have decreased if they were not treated with patching. The absence of a control group of children not exposed to patching limits the strength of our study. However, it is not ethical to withhold therapy in situations where it has been shown to help.

Functional amblyopia overlying the organic cause of visual loss may be compounded by the development of secondary strabismus, as seen in four of our patients. In one case series, ${ }^{7}$ the final visual acuity recorded in eyes with macular retinoblastoma varied between 0.1 logMAR to fixation responses recorded as not central, unsteady, or unmaintained. In this series visual acuity was recorded in three of the affected eyes with only a fixation response in younger patients. In two patients treated for foveal tumors, a visual acuity of 0.1 $\log$ MAR was measured. Another study ${ }^{14}$ reported visual acuities ranging from 20/100 to hand movements in seven patients with macular retinoblastoma followed up for 12-17 years. Nine of 19 patients with posterior pole tumors $(47.3 \%)$ were reported to have achieved a visual acuity of 6/60 (1.0 $\log$ MAR) or better. ${ }^{3}$ None of these studies mentioned therapy with active patching. Lueder et al ${ }^{10}$ reported a child with foveal retinoblastoma who developed normal visual acuity following laser hyperthermia and chemotherapy complemented by active occlusion therapy, despite initial resistance to it.

In our study all children who did not comply with patching showed no improvement in visual acuity. In one of these children the tumor did not directly 


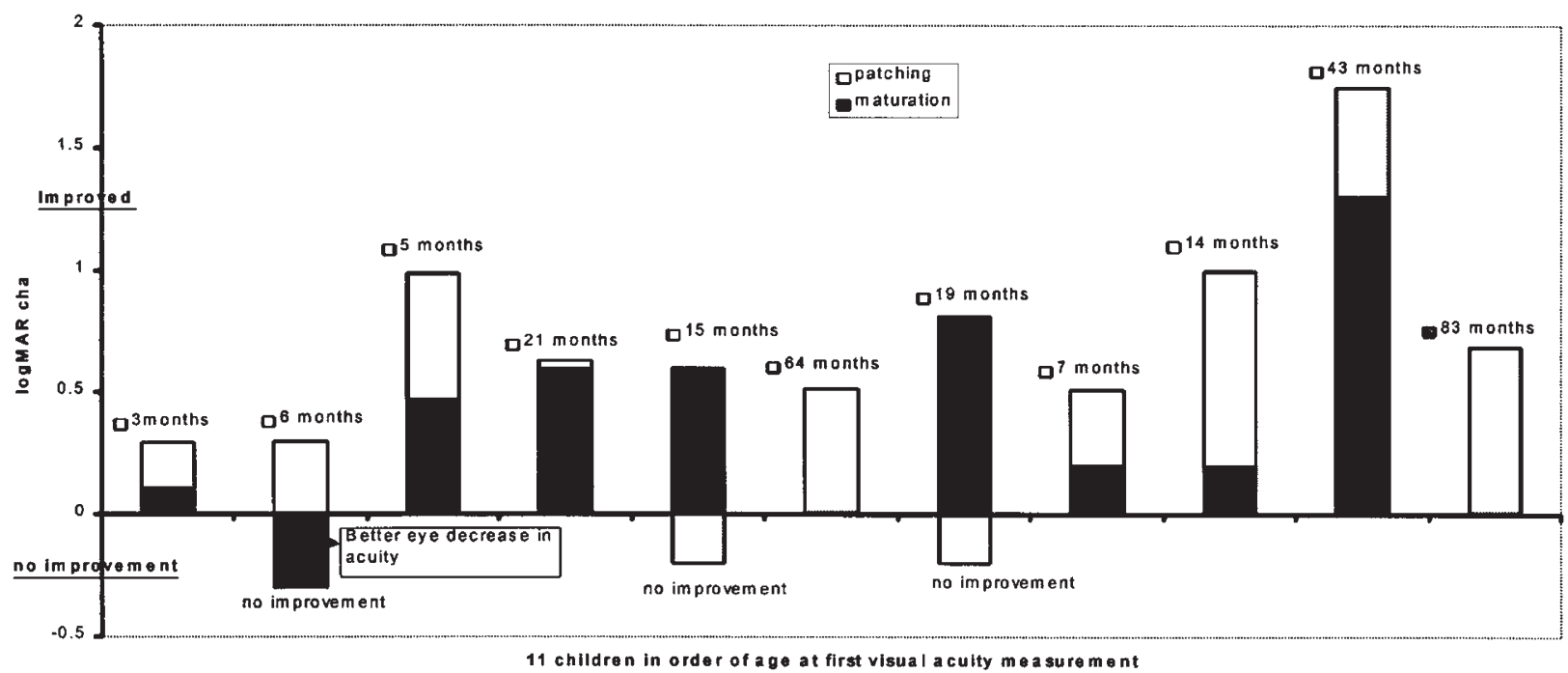

Figure 4 Histogram of 11 children demonstrating the possible contribution of visual maturation in the final visual acuity assessment. Each bar represents a child, and above it is the number of months occlusion therapy was carried out. Bars 2, 5 and 7 represent the children who showed no improvement in visual acuity. In bar 2 the child developed poorer vision in the control eye.

involve the fovea. Our study found no direct correlation between response to therapy and foveal involvement. In one study, ${ }^{9}$ visual outcome based on the appearance and location of the tumor could be predicted in only $54 \%$ of children with retinoblastoma, suggesting that many children with two eyes, one with macular involvement, might benefit from occlusion therapy.

The present study demonstrated an overall benefit of amblyopia therapy for children with retinoblastoma and two eyes when the macula is involved in one eye. Our small sample size did not permit us to correlate age, patching, and foveal involvement with visual improvement. Since younger patients are still receiving amblyopia therapy, further improvement may occur.

Given the current modalities of treatment for retinoblastoma, the prognosis for survival is excellent, and an increasing number of eyes with retinoblastoma are salvaged by treatment. Eyes with posterior pole tumors in patients with two eyes may have a coexisting functional amblyopia. Occlusion therapy and a close follow-up of the visual acuity in both eyes can result in improved vision despite an anatomic macular deficit. Parents are willing to pursue patching when they understand its importance in optimizing the potential of their child's eyes.

\section{Acknowledgements}

This paper was prepared with assistance of Editorial Services, The Hospital for Sick Children, Toronto, Canada. P Watts was supported by Pharmacia Upjohn.

\section{References}

1 Murphree AL. Retinoblastoma. In: Traboulsi E (ed). Genetic Diseases of the Eye. Oxford University Press: Oxford, 1998, pp 813-849.

2 Sanders BM, Draper GJ, Kingston JE. Retinoblastoma in Great Britain 1969-1980: incidence treatment and survival. Br J Ophthalmol 1988; 72: 576-583.

3 Migdal C. Bilateral retinoblastoma: the prognosis for vision. Br J Ophthalmol 1983; 67: 592-595.

4 Gallie BL, Budning A, DeBoer G, Thiessen JJ, Koren G, Verjee $\mathrm{Z}$ et al. Chemotherapy with focal therapy can cure intraocular retinoblastoma without radiotherapy. Arch Ophthalmol 1996; 114: 1321-1328.

5 Murphree AL, Villablanca JG, Deegan WF, Sato JK, Malogolowkin M, Fisher A et al. Chemotherapy plus local treatment in the management of intraocular retinoblastoma. Arch Ophthalmol 1996; 114: 1348-1356.

6 Kingston JE, Hungerford JL, Madreperla SA, Plowman PN. Results of combined chemotherapy and radiotherapy for advanced intraocular retinoblastoma. Arch Ophthalmol 1996; 114: 1339-1343.

7 Lam BL, Judisch GF, Sobol WM, Blodi CF. Visual prognosis in macular retinoblastomas. Am J Ophthalmol 1990; 110: 229-232.

8 Weiss AV, Karr DJ, Kalina RE, Lindsley KL, Pendergrass TW. Visual outcomes of macular retinoblastoma after external beam radiation therapy. Ophthalmology 1994; 101: 1244-1249.

9 Hall LS, Ceisler E, Abramson DH. Visual outcomes in children with bilateral retinoblastoma. JAAPOS 1999; 3: 138-142.

10 Lueder GT, Goyal R. Visual function after laser hyperthermia and chemotherapy for macular retinoblastoma. Am J Ophthalmol 1996; 121: 582-584.

11 Lang LLH, Lambert SR. Reappraisal of occlusion therapy for severe structural abnormalities of the optic disc and macula. J Pediatr Ophthalmol Strabismus 1995; 32: 37-41. 
12 Kushner BJ. Functional amblyopia associated with organic ocular disease. Am J Ophthalmol 1981; 91: 39-45.

13 Simons Kurt, Preslan M. Natural history of amblyopia untreated owing to lack of compliance. Br J Ophthalmol 1999; 83: 582-587.
14 Holbeck S, Ehlers N. Long term visual results in eyes cured for retinoblastoma by radiation. Acta Ophthalmol 1989; 67: 560. 